\title{
Antimicrobial Susceptibility of Gram-Positive Bacteria Isolated in Brazilian Hospitals Participating in the SENTRY Program (2005-2008)
}

\begin{abstract}
Ana C. Gales ${ }^{1}$, Helio S. Sader ${ }^{1,2}$, Julival Ribeiro ${ }^{3}$, Cassia Zoccoli ${ }^{4}$, Afonso Barth ${ }^{5}$ and Antonio C. Pignatari ${ }^{1}$ ${ }^{1}$ Universidade Federal de São Paulo, São Paulo, SP, Brazil; ${ }^{2} J M I$ Laboratories, North Liberty, IA, USA; ${ }^{3}$ Hospital de Base do Distrito Federal, Brasília, DF, Brazil; ${ }^{4}$ Laboratório Médico Santa Luzia, Florianópolis, SC, Brazil; ${ }^{5}$ Hospital de Clínicas de Porto Alegre, Porto Alegre, RS, Brazil
\end{abstract}

We report the antimicrobial susceptibility patterns of the most frequently isolated Gram-positive bacteria in the Brazilian hospitals participating in the SENTRY Antimicrobial Surveillance Program. The strains were consecutively collected (one per patient) between January 2005 and September 2008 and susceptibility tested by reference broth microdilution methods at the JMI Laboratories (North Liberty, Iowa, USA). A total of 3,907 Gram-positive cocci were analyzed. The Gram-positive organisms most frequently isolated from bloodstream infections were Staphylococcus aureus (2,218 strains; $20.2 \%$ of total), coagulase-negative staphylococci (CoNS; 812 strains [14.7\%]), and Enterococcus spp. (754 strains; $5.0 \%)$. S. aureus ranked first (28.1\%) and Enterococcus faecalis ranked $7^{\text {th }}(4.5 \%)$ among cases of skin and soft tissue infections. $S$. aureus was also the second most frequently isolated pathogen from patients with lower respiratory tract infections (24.9\% of cases) after Pseudomonas aeruginosa $(30.5 \%)$. Resistance to oxacillin was observed in $31.0 \%$ of $S$. aureus and the vast majority of oxacillin-resistant (MRSA) strains were also resistant to clindamycin, ciprofloxacin and levofloxacin. Vancomycin, linezolid and daptomycin were all very active against $S$. aureus strains tested (>99.9-100.0\% susceptible), but daptomycin $\left(\mathrm{MIC}_{50}, 0.25 \mu \mathrm{g} / \mathrm{mL}\right.$ and $\mathrm{MIC}_{90}, 0.5 \mu \mathrm{g} / \mathrm{mL}$ ) was four- to eight-fold more potent than vancomycin ( $\mathrm{MIC}_{50}$ and $\mathrm{MIC}_{90}$ of $1 \mu \mathrm{g} / \mathrm{mL}$ ) and linezolid (MIC $\mathrm{M0}_{50} 1 \mu \mathrm{g} / \mathrm{mL}$ and $\left.\mathrm{MIC}_{90}, 2 \mu \mathrm{g} / \mathrm{mL}\right)$. Vancomycin resistance increased significantly among enterococci during the study period, but it was restrict to only one medical center until 2007 and emerged in a second medical center in 2008. Daptomycin was the most active antimicrobial tested against enterococci in general $(\mathbf{1 0 0 . 0} \%$ susceptible), followed by linezolid (99.9\% susceptible), ampicillin (87.4\%) and vancomycin (84.6\%). In conclusion, daptomycin and linezolid showed excellent in vitro activity against contemporary Gram-positive organisms $(3,907)$ collected in Brazilian hospitals monitored by the SENTRY Program, including MRSA, vancomycin-resistant enterococci (VRE) and other multidrugresistant organisms. Although vancomycin resistance rates in Brazil appears to be relatively low compared to those reported in the USA, VRE has emerged and rapidly disseminated in some Brazilian medical centers.

Key-Words: Antimicrobial resistance, SENTRY, nosocomial infections, Brazil.

Among the Gram-positive bacteria, staphylococci, streptococci and enterococci are important causes of both community- and hospital-acquired infections. Staphylococcus aureus is particularly important as a frequent cause of sepsis and many other types of nosocomial-acquired infections. This organism represents the first or second most frequently isolated pathogen from bloodstream infections, skin and skin structure infections (SSSI), and pneumonia in hospitalized patients [1,2]. Although the prevalence of methicillin-resistant S. aureus (MRSA) may vary significantly, it is usually high in Brazilian hospitals, especially in intensive care units (ICU). Furthermore, MRSA are usually resistant to most antimicrobial agents available for clinical use [3,4].

Coagulase-negative staphylococci (CoNS) has being also recognized as an important cause of nososcomial infections and this organism is usually more resistant to antimicrobial agents than $S$. aureus [5]; while Enterococcus spp., mainly E. faecalis and $E$. faecium, are among the most frequently isolated pathogens from nosocomial infections in the United States

Received on 26 July 2008; revised 12 December 2008.

Address for correspondence: Dr. Helio S. Sader, M.D., Ph.D. JMI Laboratories, 345 Beaver Kreek Centre, Suite A, North Liberty Iowa 52317. Phone: (319) 665-3370. Fax: (319) 655-3371. E-mail: heliosader@jmilabs.com.

The Brazilian Journal of Infectious Diseases

2009;13(2):90-98. (C) 2009 by The Brazilian Journal of Infectious Diseases and Contexto Publishing. All rights reserved.
(USA) [1]. Vancomycin-resistant enterococci (VRE) emerged in the early 80's in USA hospitals and rapidly disseminated throughout the country [6]. More recently, the occurrence of VRE is increasing in many European hospitals while data from Latin American countries are still scarce [7]

The SENTRY Antimicrobial Surveillance Program was designed to monitor antimicrobial resistance among various types of infection $[2,8,9]$. The Program was initiated in early 1997 and today it includes more than 120 medical centers in North America, South America, Europe, Asia, and Western Pacific regions. Data generated by large multicenter programs are of great importance especially in developing regions such as Latin America, where extensive surveillance studies are not routinely conducted. We report the antimicrobial susceptibility of the most frequently isolated Gram-positive cocci in the Brazilian hospitals that participated in the SENTRY Program in the 2005-2008 period.

\section{Material and Methods}

In Brazil, four institutions participate in the SENTRY Program: Hospital São Paulo / UNIFESP, São Paulo, SP (A.C. Gales, A.C. Pignatari and S. Andrade), Hospital de Clínicas de Porto Alegre, Porto Alegre, RS (A. Barth), Hospital de Base do Distrito Federal, Brasília, DF (J. Ribeiro) and Laboratório Médico Santa Luzia, Florianópolis, SC (C. Zoccoli) which collects bacterial isolates from 4 regional smaller public and/ or private hospitals (40 to 240 beds). 
Each institution collects approximately 500 consecutive, non-duplicate bacterial isolates every year. All isolates are identified at the participating institution by routine methodologies in use at each laboratory. Upon receipt at the central monitor (JMI Laboratories, North Liberty, IA, USA), isolates were subcultured to ensure viability and purity. Confirmation of species identification was performed with the Vitek system (bioMérieux Vitek, St Louis, MO) or conventional methods, as required.

A total of 3,907 Gram-positive bacteria collected between January 2005 and September 2008, were analyzed in the present study. The organisms were consecutively collected according to the type of infections, which included mainly bloodstream infections (57\% of strains), skin and skin structure infections $(17 \%)$ and pneumonia in hospitalized patients $(10 \%)$. The Gram-negative organisms were analyzed separately and results reported in another publication [9]. The organism collection evaluated in this study included S. aureus (2,218 strains), CoNS (812 strains), Enterococcus spp. (754 strains), $\beta$ haemolytic streptococci (99 strains) and viridians group streptococci (24 strains).

Antimicrobial susceptibility testing was performed by the broth microdilution method, following recommendations of the Clinical and Laboratory Standards Institute [10]. Antimicrobial powders were obtained from the respective manufacturers and microdilution plates were prepared by TREK Diagnostics (Cleveland, OH, USA). Susceptibility results were interpreted according to CLSI document M100S18 [11]. Quality control was performed by testing S. aureus ATCC 29213, E. faecalis ATCC 29212 and Streptococcus pneumoniae ATCC 49619.

\section{Results}

Approximately $60 \%$ of isolates evaluated in the present study were from bloodstream infections and the frequency of occurrence of organisms isolated from this type of infection is summarized in Figure 1. S. aureus (20.2\% of total) was the most common cause of bloodstream infection, followed by CoNS (14.5\%). Enterococcus spp. ranked $8^{\text {th }}$ and was isolated from $5.0 \%$ of bloodstream infection cases. S. aureus was also the most common cause of SSSI (28.1\%; Figure 2) and was isolated from $24.9 \%$ of patients with pneumonia (Figure 3). Enterococcus spp. was responsible for $4.5 \%$ of SSSI cases (Figure 2).

In general, $31.0 \%$ of $S$. aureus strains were resistant to oxacillin (MRSA) and the vast majority of MRSA strains were also resistant to clindamycin, ciprofloxacin and levofloxacin (Table 1). Furthermore, $68.1 \%$ of MRSA strains were resistant to trimethoprim/sulfamethoxazole. Daptomycin and vancomycin were active against all $S$. aureus strains tested (100.0\% susceptible). Linezolid was also very active against S. aureus with only one strain being non-susceptible (MIC, 8 $\mu \mathrm{g} / \mathrm{mL}$ ) to this antimicrobial. Daptomycin ( $\mathrm{MIC}_{50}, 0.25 \mu \mathrm{g} / \mathrm{mL}$ and $\mathrm{MIC}_{90}, 0.5 \mu \mathrm{g} / \mathrm{mL}$ ) was four- to eight-fold more potent than vancomycin ( $\mathrm{MIC}_{50}$ and $\mathrm{MIC}_{90}$ of $1 \mu \mathrm{g} / \mathrm{mL}$ ) and linezolid
Figure 1. Frequency of occurrence of pathogens causing bloodstream infections in Brazilian hospitals (3,807 strains; 2005-2008).

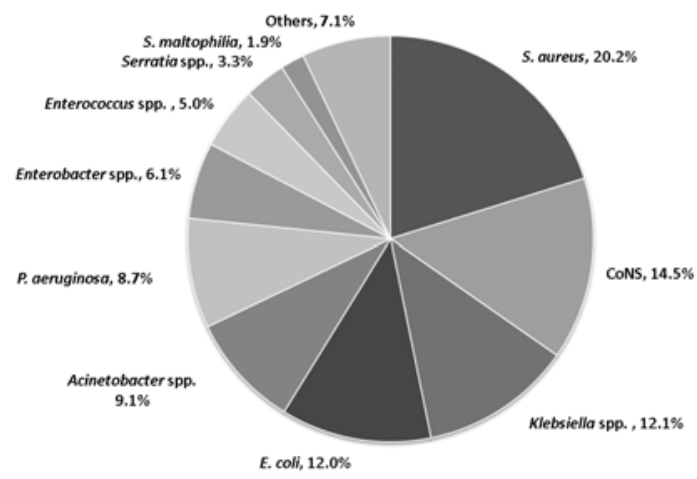

Figure 2. Frequency of occurrence of pathogens causing skin and soft tissue infections in Brazilian hospitals (605 strains; 2005-2008).

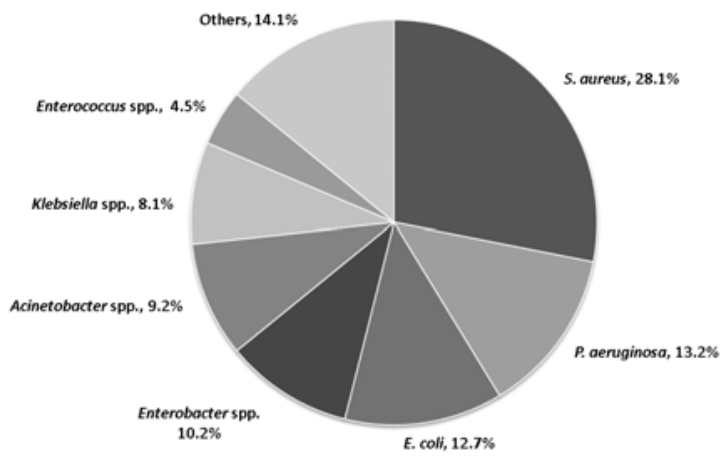

Figure 3. Frequency of occurrence of pathogens isolated from patients hospitalized with pneumonia in Brazilian hospitals (875 strains; 2005-2008).

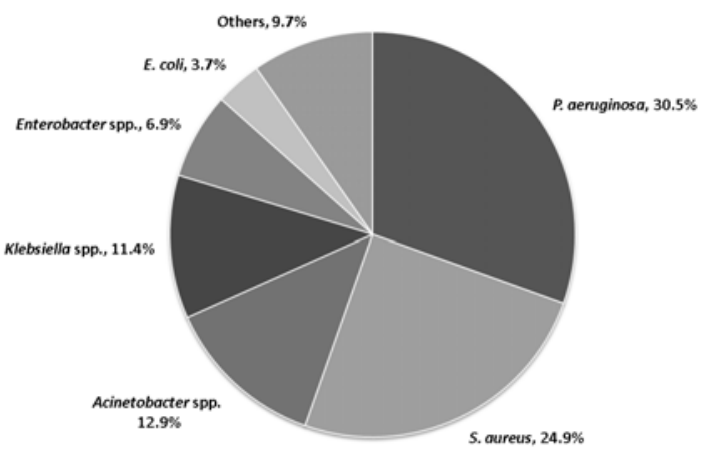


Table 1. Antimicrobial susceptibility of staphylococci isolated in Brazilian hospitals (2005-2008).

\begin{tabular}{|c|c|c|c|c|}
\hline \multirow{2}{*}{$\begin{array}{l}\text { Organism (no. tested)/ } \\
\text { Antimicrobial agent }\end{array}$} & \multicolumn{2}{|c|}{ MIC $(\mu \mathrm{g} / \mathrm{mL})$} & \multirow[t]{2}{*}{$\%$ susceptible $^{\mathrm{a}}$} & \multirow[t]{2}{*}{$\%$ resistant $^{\mathrm{a}}$} \\
\hline & $50 \%$ & $90 \%$ & & \\
\hline \multicolumn{5}{|l|}{$\overline{\operatorname{MSSA}}(1,531)$} \\
\hline Erythromycin & $\leq 0.25$ & $>2$ & 86.2 & 13.0 \\
\hline Clindamycin & $\leq 0.25$ & $\leq 0.25$ & 98.1 & 1.7 \\
\hline Ciprofloxacin & 0.25 & 0.5 & 95.2 & 2.2 \\
\hline Levofloxacin & $\leq 0.5$ & $\leq 0.5$ & 97.8 & 2.0 \\
\hline Tetracycline & $\leq 2$ & $>8$ & 83.9 & 14.5 \\
\hline TMP/SMX & $\leq 0.5$ & $\leq 0.5$ & 98.6 & 1.4 \\
\hline Linezolid & 2 & 2 & 100.0 & $\mathrm{-b}^{\mathrm{b}}+\mathrm{c}$ \\
\hline Vancomycin & 1 & 1 & 100.0 & 0.0 \\
\hline Daptomycin & 0.25 & 0.5 & 100.0 & - \\
\hline \multicolumn{5}{|l|}{ MRSA(687) } \\
\hline Erythromycin & $>2$ & $>2$ & 6.0 & 94.0 \\
\hline Clindamycin & $>2$ & $>2$ & 11.2 & 87.9 \\
\hline Ciprofloxacin & $>4$ & $>4$ & 7.6 & 91.4 \\
\hline Levofloxacin & 4 & $>4$ & 8.6 & 90.2 \\
\hline Tetracycline & $\leq 2$ & $>8$ & 52.8 & 46.7 \\
\hline TMP/SMX & $>2$ & $>2$ & 31.9 & 68.1 \\
\hline Linezolid & 1 & 2 & 99.9 & - \\
\hline Vancomycin & 1 & 1 & 100.0 & 0.0 \\
\hline Daptomycin & 0.5 & 0.5 & 100.0 & - \\
\hline \multicolumn{5}{|l|}{ All S. aureus $(2,218)$} \\
\hline Oxacillin & 0.5 & $>2$ & 69.0 & 31.0 \\
\hline Erythromycin & $\leq 0.25$ & $>2$ & 61.3 & 38.1 \\
\hline Clindamycin & $\leq 0.25$ & $>2$ & 70.7 & 28.4 \\
\hline Ciprofloxacin & 0.5 & $>4$ & 68.1 & 29.9 \\
\hline Levofloxacin & $\leq 0.5$ & $>4$ & 70.2 & 29.3 \\
\hline Tetracycline & $\leq 2$ & $>8$ & 74.3 & 24.5 \\
\hline TMP/SMX' & $\leq 0.5$ & $>2$ & 77.9 & 22.1 \\
\hline Linezolid & 1 & 2 & $>99.9$ & - \\
\hline Vancomycin & 1 & 1 & 100.0 & 0.0 \\
\hline Daptomycin & 0.25 & 0.5 & 100.0 & - \\
\hline \multicolumn{5}{|l|}{ CoNS (812) } \\
\hline Oxacillin & $>2$ & $>2$ & 21.3 & 78.7 \\
\hline Erythromycin & $>2$ & $>2$ & 35.4 & 69.3 \\
\hline Clindamycin & $>2$ & $>2$ & 47.4 & 50.9 \\
\hline Ciprofloxacin & 4 & $>4$ & 42.2 & 55.7 \\
\hline Levofloxacin & 2 & $>4$ & 43.5 & 46.0 \\
\hline Tetracycline & $\leq 2$ & $>8$ & 82.8 & 16.0 \\
\hline $\mathrm{TMP}^{\mathrm{S} M \mathrm{MX}^{\mathrm{c}}}$ & 2 & $>2$ & 50.3 & 49.7 \\
\hline Linezolid & 1 & 1 & 99.8 & - \\
\hline Vancomycin & 1 & 2 & 100.0 & 0.0 \\
\hline Daptomycin & 0.25 & 0.5 & 99.8 & - \\
\hline
\end{tabular}

a. According to CLSI breakpoints [11]; b. - = no breakpoint has been established by the CLSI or USA-FDA; c. TMP/SMX = trimethoprim/ sulfamethoxazole.

$\left(\mathrm{MIC}_{50}, 1 \mu \mathrm{g} / \mathrm{mL}\right.$ and $\mathrm{MIC}_{90}, 2 \mu \mathrm{g} / \mathrm{mL}$ ) when tested against $S$. aureus (Table 1).

Almost $80 \%$ of CoNS strains were resistant to oxacillin. This organism showed high rates of resistance to most antimicrobial agents tested (Table 1). Vancomycin ( $\mathrm{MIC}_{50}, 1$ $\mu \mathrm{g} / \mathrm{mL}$ and $\mathrm{MIC}_{90}, 2 \mu \mathrm{g} / \mathrm{mL}$ ) was active against all CoNS strain at the susceptible breakpoint while two strains $(0.2 \%)$ showed decreased susceptibility to daptomycin (MIC of $2 \mu \mathrm{g} / \mathrm{mL}$, one doubling dilution above the susceptible breakpoint) and two other strains showed high level of resistance to linezolid (MIC, $>8 \mu \mathrm{g} / \mathrm{mL}$ ).

Approximately $83 \%$ of enterococci were E. faecalis and 7.7\% showed vancomycin resistance (Table 2). Daptomycin $\left(\mathrm{MIC}_{50}\right.$ and $\mathrm{MIC}_{90}$ of $1 \mu \mathrm{g} / \mathrm{mL}$ ) was active against all E. faecalis 
strains (100.0\% susceptible) while linezolid ( $\mathrm{MIC}_{50}, 1 \mu \mathrm{g} / \mathrm{mL}$ and $\mathrm{MIC}_{90}, 2 \mu \mathrm{g} / \mathrm{mL}$ ) was active against 99.8\%, ampicillin $\left(\mathrm{MIC}_{50}, \leq 1 \mu \mathrm{g} / \mathrm{mL}\right.$ and $\mathrm{MIC}_{90}, 4 \mu \mathrm{g} / \mathrm{mL}$ ) was active against 98.9\% and teicoplanin ( $\mathrm{MIC}_{50}$ and $\mathrm{MIC}_{90}$ of $\leq 2 \mu \mathrm{g} / \mathrm{mL}$ ) inhibited $92.3 \%$ of strains at the susceptible breakpoint.

Vancomycin resistance increased from 4.4\% in 2005 to $12.2 \%$ in 2008 among $E$. faecalis and $94 \%$ of vancomycinresistant strains (46 of 49) were isolated in one medical center. On this particular medical center, vancomycin resistance increased from $30.4 \%$ in 2005 to $50.0 \%$ in 2008 among $E$. faecalis. All vancomycin-resistant $E$. faecalis strains were susceptible to daptomycin and linezolid, and 95.9\% were susceptible to ampicillin. Daptomycin was the most potent agent tested against vancomycin-resistant $E$. faecalis $\left(\mathrm{MIC}_{50}\right.$ and $\mathrm{MIC}_{90}$ of $1 \mu \mathrm{g} / \mathrm{mL}$; Table 2 ).

Among E. faecium strains, $65.7 \%$ of strains were resistant to vancomycin (Table 2). Again, the vast majority of vancomycin-resistant strains (47 of 59; 80\%) were from one medical center. Vancomycin-resistant E. faecium was not observed in any medical center in 2005 and in only one medical center in 2006 (at a rate of $74.2 \%$ ) and 2007 (68.4\%). In 2008 vancomycin-resistant $E$. faecium emerged in a second medical center and both medical centers had high rates of vancomycinresistance among $E$. faecium strains (64.7 and 78.6\%; data not shown). Only daptomycin was active against all vancomycinresistant strains. Linezolid was also very active against vancomycin-resistant $E$. faecium (98.5\% susceptible), while quinupristin/dalfopristin was active against $92.5 \%$ of strains. Although high-level streptomycin resistance was observed in $94.0 \%$ of vancomycin-resistant $E$. faecium strains, only $9.0 \%$ showed high-level resistance to gentamicin. In contrast, streptomycin resistance was observed in $16.3 \%$ of vancomycin-resistant $E$. faecalis strains, while $63.5 \%$ of these strains showed high-level resistance to gentamicin (Table 2).

When all enterococci strains are analyzed together, daptomycin was the most active antimicrobial (100.0\% susceptible), followed by linezolid (99.9\% susceptible), ampicillin (87.4\%) and vancomycin (84.6\%). Although ampicillin and vancomycin showed reasonable activity against enterococci in general (84.6\%-87.4\% susceptible), these compounds showed limited activity against E. faecium (15.7 and 34.3\% susceptible respectively; Table 2).

Among viridans group streptococci, 87.5 and $91.7 \%$ were susceptible to penicillin and ceftriaxone respectively, while daptomycin $\left(\mathrm{MIC}_{50}, 0.12 \mu \mathrm{g} / \mathrm{mL}\right.$ and $\mathrm{MIC}_{90}, 0.5 \mu \mathrm{g} / \mathrm{mL}$ ), levofloxacin $\left(\mathrm{MIC}_{50}, \leq 0.5 \mu \mathrm{g} / \mathrm{mL}\right.$ and $\mathrm{MIC}_{90}, 1 \mu \mathrm{g} / \mathrm{mL}$ ), linezolid $\left(\mathrm{MIC}_{50}\right.$ and $\mathrm{MIC}_{90}$ of $1 \mu \mathrm{g} / \mathrm{mL}$ ) and vancomycin $\left(\mathrm{MIC}_{50}\right.$ and $\mathrm{MIC}_{90}$ of $\left.0.5 \mu \mathrm{g} / \mathrm{mL}\right)$ were active against all strains tested $(100 \%$ susceptible; Table 3). $\beta$-haemolytic streptococci exhibited high rates of susceptibility to all antimicrobial agents tested, except tetracycline (27.3\% susceptible) and erythromycin (92.3\% susceptible; Table 3).

Table 4 shows the comparison of the in vitro potency of the most active compounds, vancomycin linezolid and daptomycin, tested against staphylococci and enterococci.
Daptomycin was generally four-fold more potent than linezolid and vancomycin against staphylococci (S. aureus and CoNS). All S. aureus and 99.8\% of CoNS were inhibited at daptomycin MIC of $1 \mu \mathrm{g} / \mathrm{mL}$ or less. Daptomycin and linezolid showed similar in vitro activity against enterococci while vancomycin exhibited more limited in vitro activity against this organism, especially E. faecium (only 34.3\% susceptible). MRSA strains exhibited daptomycin MIC values slightly higher (MIC $_{50}, 0.5$ $\mu \mathrm{g} / \mathrm{mL}$ ) than MSSA strains ( MIC $_{50}, 0.25 \mu \mathrm{g} / \mathrm{mL}$; Table 5). In contrast, vancomycin-susceptible and -resistant enterococci showed similar susceptibility to vancomycin (Table 5). CoNS $\left(\mathrm{MIC}_{50}, 0.25 \mu \mathrm{g} / \mathrm{mL}\right.$ ), as well as viridans group and $\beta$-haemolytic streptococci $\left(\mathrm{MIC}_{50}, \leq 0.12 \mu \mathrm{g} / \mathrm{mL}\right)$ showed very low daptomycin MIC values (Table 5).

\section{Discussion}

The high prevalence of MRSA in some Brazilian hospitals is a concern because these isolates are often resistant to multiple antimicrobial agents. The overall MRSA rate in the present study was $31.0 \%$, which is comparable to that reported in previous studies [2]. However, MRSA rates may vary greatly among hospitals or even among units of a hospital. The emergence of MRSA is largely due to dissemination of clonal strains, and temporary hospital outbreaks are typically due to cross-transmission between patients of these strains. Furthermore, a direct correlation between antimicrobial usage and resistant rates has been difficult to establish due to a high number of variables involved [12,13].

Vancomycin-resistant $S$. aureus (VRSA) is a serious concern, but only very few isolates have been reported, all from the USA and most of them from the state of Michigan. In contrast, there are many reports of vancomycin-intermediate S. aureus (VISA), especially after the reduction of the CLSI vancomycin-susceptible breakpoint from 4 to $2 \mu \mathrm{g} / \mathrm{mL}$ [14]. Interestingly, we did not observe any $S$. aureus isolate with a vancomycin-intermediate MIC value ( $4 \mu \mathrm{g} / \mathrm{mL})$ in the present study.

Another interesting finding of this study was the documented linezolid resistance (one strain with MIC of $8 \mu \mathrm{g} /$ $\mathrm{mL}$ ) and quinupristin/dalfopristin resistance (two strains with MIC of $2 \mu \mathrm{g} / \mathrm{mL}$ [intermediate] and one strain with MIC $>2 \mu \mathrm{g} /$ $\mathrm{mL}$ ) among $S$. aureus. Although linezolid-resistant $S$. aureus has been previously reported from Brazil [15], it remains extremely rare in the Brazilian hospitals monitored by the SENTRY Program. Acquired quinupristin/dalfopristin resistance was reported in E. faecium from Brazilian hospitals before this antimicrobial became available for clinical use in this country [16]. The emergence and dissemination of this resistance phenotype may be related to the clinical use of natural streptogramin mixtures such as pristiniamycin and synergistin, orally and topically since the 1960s.

S. aureus was the most common Gram-positive organism recovered from bloodstream infections (20.2\% of cases) and SSSI (28.1\%), and the second most common from patients with pneumonia (24.9\%). Furthermore, $31.0 \%$ of strains were 
Table 2. Antimicrobial susceptibility of enterococci isolated in Brazilian hospitals (2005-2008).

\begin{tabular}{|c|c|c|c|c|}
\hline \multirow{2}{*}{$\begin{array}{l}\text { Organism (no. tested)/ } \\
\text { Antimicrobial agent }\end{array}$} & \multicolumn{2}{|c|}{ MIC $(\mu \mathrm{g} / \mathrm{mL})$} & \multirow[t]{2}{*}{$\%$ susceptible ${ }^{a}$} & \multirow[t]{2}{*}{$\%$ resistant $^{\mathrm{a}}$} \\
\hline & $50 \%$ & $90 \%$ & & \\
\hline \multicolumn{5}{|l|}{ E. faecalis } \\
\hline \multicolumn{5}{|l|}{ Vancomycin-susceptible. (576) } \\
\hline Ampicillin & $\leq 1$ & 4 & 99.1 & 0.0 \\
\hline Levofloxacin & 1 & $>4$ & 67.4 & 31.6 \\
\hline Gentamicin (HL) ${ }^{c}$ & $\leq 500$ & $>100$ & 74.3 & 25.7 \\
\hline Streptomycin (HL) & $\leq 1000$ & $>200$ & 74.0 & 26.0 \\
\hline Linezolid & 1 & 2 & 99.8 & 0.2 \\
\hline Teicoplanin & $\leq 2$ & $\leq 2$ & 100.0 & 0.0 \\
\hline Daptomycin & 1 & 1 & 100.0 & $-\mathrm{b}$ \\
\hline \multicolumn{5}{|l|}{ Vancomycin-resistant (49) } \\
\hline Ampicillin & 2 & 8 & 95.9 & 4.1 \\
\hline Levofloxacin & $>4$ & $>4$ & 4.1 & 95.9 \\
\hline Gentamicin (HL) & $>1000$ & $>1000$ & 26.5 & 63.5 \\
\hline Streptomycin (HL) & $\leq 1000$ & $>2000$ & 83.7 & 16.3 \\
\hline Linezolid & 1 & 2 & 100.0 & 0.0 \\
\hline Teicoplanin & $>16$ & $>16$ & 2.0 & 98.0 \\
\hline Daptomycin & 0.5 & 1 & 100.0 & - \\
\hline \multicolumn{5}{|l|}{ All E. faecalis (625) } \\
\hline Ampicillin & $\leq 1$ & 4 & 98.9 & 1.1 \\
\hline Levofloxacin & 1 & $>4$ & 62.4 & 36.6 \\
\hline Gentamicin (HL) & $\leq 500$ & $>1000$ & 70.6 & 29.4 \\
\hline Streptomycin (HL) & $\leq 1000$ & $>2000$ & 74.7 & 25.3 \\
\hline Linezolid & 1 & 2 & 99.8 & 0.2 \\
\hline Teicoplanin & $\leq 2$ & $\leq 2$ & 92.3 & 7.7 \\
\hline Vancomycin & 2 & 2 & 92.2 & 7.7 \\
\hline Daptomycin & 1 & 1 & 100.0 & - \\
\hline \multicolumn{5}{|l|}{ E. faecium } \\
\hline \multicolumn{5}{|l|}{ Vancomycin-susc. (35) } \\
\hline Ampicillin & $>16$ & $>16$ & 45.7 & 52.3 \\
\hline Levofloxacin & 4 & $>4$ & 48.6 & 42.9 \\
\hline Gentamicin(HL) & $\leq 500$ & $>1000$ & 82.9 & 17.1 \\
\hline Streptomycin (HL) & $\leq 1000$ & $>2000$ & 54.3 & 45.7 \\
\hline Quinupristin/dalfopristin & 1 & $>2$ & 57.1 & 20.0 \\
\hline Linezolid & 1 & 2 & 100.0 & 0.0 \\
\hline Teicoplanin & $\leq 2$ & $\leq 2$ & 100.0 & 0.0 \\
\hline Daptomycin & 2 & 4 & 100.0 & - \\
\hline \multicolumn{5}{|l|}{ Vancomycin-resistant (67) } \\
\hline Ampicillin & $>16$ & $>16$ & 0.0 & 100.0 \\
\hline Levofloxacin & $>4$ & $>4$ & 0.0 & 98.5 \\
\hline Gentamicin (HL) & $\leq 500$ & $\leq 500$ & 91.0 & 9.0 \\
\hline Streptomycin (HL) & $>2000$ & $>2000$ & 6.0 & 94.0 \\
\hline Quinupristin/dalfopristin & 1 & 1 & 92.5 & 3.0 \\
\hline Linezolid & 1 & 2 & 98.5 & 1.5 \\
\hline Teicoplanin & $>16$ & $>16$ & 0.0 & 98.5 \\
\hline Daptomycin & 2 & 2 & 100.0 & -0 \\
\hline \multicolumn{5}{|l|}{ All E. faecium (102) } \\
\hline Ampicillin & $>16$ & $>16$ & 15.7 & 84.3 \\
\hline Levofloxacin & $>4$ & $>4$ & 16.7 & 79.4 \\
\hline Gentamicin (HL) & $\leq 500$ & $>1000$ & 88.2 & 11.8 \\
\hline Streptomycin (HL) & $>1000$ & $>1000$ & 22.6 & 77.4 \\
\hline
\end{tabular}




\begin{tabular}{lllll}
\hline Quinupristin/dalfopristin & 1 & 2 & 80.4 & 8.8 \\
Linezolid & 1 & 2 & 99.0 & 1.0 \\
Teicoplanin & $>16$ & $>16$ & 34.3 & 64.7 \\
Vancomycin & $>16$ & $>16$ & 34.3 & 65.7 \\
Daptomycin & 2 & 2 & 100.0 & - \\
& & & & \\
All enterococi (754) ${ }^{\mathrm{d}}$ & & & 87.4 & 12.6 \\
Ampicillin & $\leq 1$ & $>16$ & 56.6 & 41.9 \\
Levofloxacin & 2 & $>4$ & 73.3 & 26.7 \\
Gentamicin(HL) & $\leq 500$ & $>1000$ & 67.9 & 32.1 \\
Streptomycin (HL) & $\leq 1000$ & $>2000$ & 12.3 & 80.8 \\
Quinupristin/dalfopristin & $>2$ & $>2$ & 99.9 & 0.1 \\
Linezolid & 1 & 2 & 84.6 & 15.3 \\
Teicoplanin & $\leq 2$ & $>16$ & 84.3 & 15.4 \\
Vancomycin & 2 & $>16$ & 100.0 & - \\
Daptomycin & 1 & 1 & \\
\hline
\end{tabular}

a. According to CLSI breakpoints [11]; b.- = no breakpoint has been established by the CLSI or USA-FDA; c. HL = high level resistance; d. Includes E. faecalis (625), E. faecium (102), E. avium (9), E. gallinarum (4), E. hirae (2), E. durans (1) and Enterococcus spp. (11).

Table 3. Antimicrobial susceptibility of streptococci isolated in Brazilian hospitals (2005-2008).

\begin{tabular}{|c|c|c|c|c|}
\hline \multirow{2}{*}{$\begin{array}{l}\text { Organism (no. tested)/ } \\
\text { Antimicrobial agent }\end{array}$} & \multicolumn{2}{|c|}{$\operatorname{MIC}(\mu \mathrm{g} / \mathrm{mL})$} & \multirow{2}{*}{ \% susceptible } & \multirow{2}{*}{$\%$ resistant } \\
\hline & $50 \%$ & $90 \%$ & & \\
\hline \multicolumn{5}{|c|}{ Viridans group streptococci (24) } \\
\hline Penicillin & 0.03 & 0.25 & 87.5 & 4.2 \\
\hline Ceftriaxone & $\leq 0.25$ & 0.5 & 91.7 & 8.3 \\
\hline Erythromycin & $\leq 0.25$ & 2 & 62.5 & 37.5 \\
\hline Clindamycin & $\leq 0.25$ & $\leq 0.25$ & 95.8 & 4.2 \\
\hline Levofloxacin & $\leq 0.5$ & 1 & 100.0 & 0.0 \\
\hline Tetracycline & $\leq 2$ & $>8$ & 83.3 & 16.7 \\
\hline Linezolid & 1 & 1 & 100.0 & $-\mathrm{b}$ \\
\hline Vancomycin & 0.5 & 0.5 & 100.0 & 0.0 \\
\hline Daptomycin & 0.12 & 0.5 & 100.0 & - \\
\hline \multicolumn{5}{|c|}{$\beta$-haemolytic streptococci (99) } \\
\hline Penicillin & 0.03 & 0.06 & 100.0 & 0.0 \\
\hline Ceftriaxone & 0.25 & 0.25 & 100.0 & 0.0 \\
\hline Erythromycin & $\leq 0.25$ & $\leq 0.25$ & 92.9 & 7.1 \\
\hline Clindamycin & $\leq 0.25$ & $\leq 0.25$ & 98.0 & 2.0 \\
\hline Levofloxacin & $\leq 0.25$ & 1 & 100.0 & 0.0 \\
\hline Tetracycline & $>8$ & $>8$ & 27.3 & 72.7 \\
\hline Trimethoprim/sulfa & $\leq 0.5$ & $=0.5$ & 99.0 & 1.0 \\
\hline Linezolid & 1 & 1 & 100.0 & - \\
\hline Vancomycin & 0.5 & 0.5 & 100.0 & 0.0 \\
\hline Daptomycin & 0.12 & 0.25 & 100.0 & - \\
\hline
\end{tabular}

resistant to oxacillin. This finding emphasizes the importance of the inclusion of an anti-MRSA drug in the initial antimicrobial regimen for these infections [18,19]. Three antimicrobial agents exhibited excellent potency and spectrum against MRSA and staphylococci in general: vancomycin, linezolid and daptomycin (Table 1). Vancomycin has been the standard antimicrobial therapy for serious MRSA infections since the early 1980s, when MRSA emerged as a significant nosocomial pathogen in the USA [20]. However, vancomycin has demonstrated slower in vitro bactericidal activity and clinical responses compared with antistaphylococcal beta-lactams. A reduction of efficacy of vancomycin against vancomycinsusceptible MRSA strains with elevated vancomycin MIC values $(1-2 \mu \mathrm{g} / \mathrm{mL})$ has also been extensively reported [14]. Furthermore, poor clinical response may also be related to the lack of bactericidal activity, which has been reported in approximately $20 \%$ of MRSA strains [21-24].

Linezolid also showed excellent anti-staphylococci spectrum, but this agent is predominantly bacteriostatic and has not been recommended for the treatment of some serious 
Table 4. Comparison of the in vitro potency of the most active compounds tested against Gram-positive pathogens.

\begin{tabular}{|c|c|c|c|c|c|c|c|c|c|}
\hline \multirow{3}{*}{$\begin{array}{l}\text { Organism (no. tested)/ } \\
\text { Antimicrobial agent } \\
\text { S. aureus }(2,218)\end{array}$} & \multicolumn{9}{|c|}{ No. of isolates (cumulative $\%$ ) inhibited at MIC $(\mu \mathrm{g} / \mathrm{mL})$ of: } \\
\hline & $\leq 0.12$ & 0.25 & 0.5 & 1 & 2 & 4 & 8 & 16 & $>^{\mathbf{a}}$ \\
\hline & & & & & & & & & \\
\hline Vancomycin & $1(0.1)$ & $2(0.1)$ & 293(13.4) & $1,867(97.5)$ & $55(100.0)$ & & \\
\hline Linezolid & $0(0.0)$ & $1(0.1)$ & $55(2.5)$ & 1,133(53.6) & 1,028(99.9) & $0(99.9)$ & $1(100.0)$ & $-\mathrm{b}$ & \\
\hline Daptomycin & $51(2.3)$ & $1,403(65.6)$ & 732(98.6) & $32(100.0)$ & $-\mathrm{b}$ & & & & \\
\hline \multicolumn{10}{|l|}{ CoNS (812) } \\
\hline Vancomycin & $1(0.1)$ & $5(0.7)$ & $66(8.9)$ & 444(36.6) & 290(99.3) & $6(100.0)$ & & & \\
\hline Linezolid & $3(0.4)$ & $4(0.9)$ & 209(26.6) & 569(96.7) & 25(99.8) & $0(99.8)$ & $0(99.8)$ & $-\mathrm{b}$ & $2(100.0)$ \\
\hline Daptomycin & $65(8.0)$ & 432(61.2) & 282(95.9) & $31(99.8)$ & $2(100.0)$ & & & $-\mathrm{b}$ & \\
\hline \multicolumn{10}{|l|}{ Enterococcus spp. (754) } \\
\hline Vancomycin & $0(0.0)$ & $1(0.1)$ & $38(5.2)$ & $308(46.0)$ & 283(83.6) & $6(84.4)$ & $2(84.6)$ & $0(84.6)$ & 116(100.0) \\
\hline Linezolid & $0(0.0)$ & $0(0.0)$ & $15(2.0)$ & $411(56.5)$ & 326(99.7) & $0(99.7)$ & 1(99.9) & $-\mathrm{b}$ & 1(100.0) \\
\hline Daptomycin & $6(0.8)$ & $12(2.4)$ & $305(42.8)$ & $322(85.5)$ & 100(98.8) & $9(100.0)$ & & $-\mathrm{b}$ & \\
\hline
\end{tabular}

Table 5. Antimicrobial activity of daptomycin tested against Gram-positive organisms collected in Brazilian hospitals.

\begin{tabular}{lllllll}
\hline Organism (no. tested)/ & \multicolumn{5}{l}{ No. of isolates (cumulative \%) inhibited at daptomycin MIC $(\boldsymbol{\mu g} / \mathbf{m L})$ of: } \\
\cline { 2 - 7 } Antimicrobial agent & $\mathbf{5 0 . 1 2}$ & $\mathbf{0 . 2 5}$ & $\mathbf{0 . 5}$ & $\mathbf{1}$ & $\mathbf{2}$ & $\mathbf{4}$ \\
\hline S. aureus (2,218) & $51(2.3)$ & $1403(65.6)$ & $732(98.6)$ & $32(100.0)$ & - & - \\
$\quad$ MSSA (1,531) & $41(2.7)$ & $1,211(81.8)$ & $262(98.9)$ & $17(100.0)$ & - & - \\
$\quad$ MRSA (687) & $10(1.5)$ & $192(29.4)$ & $470(97.8)$ & $15(100.0)$ & - & - \\
Enterococci spp. (754) & $6(0.8)$ & $12(2.4)$ & $305(42.8)$ & $322(855)$ & $100(98.8)$ & $9(100.0)$ \\
$\quad$ E. faecalis & & & & & & \\
$\quad$ Vancomycin-susceptible(576) & $5(0.9)$ & $7(2.1)$ & $268(48.6)$ & $261(93.9)$ & $34(99.8)$ & $1(100.0)$ \\
$\quad$ Vancomycin-resistant (49) & $0(0.0)$ & $4(8.6)$ & $21(51.0)$ & $21(93.9)$ & $2(98.0)$ & $1(100.0)$ \\
$\quad$ E. faecium & & & & & & \\
$\quad$ Vancomycin-susceptible. (35) & $0(0.0)$ & $0(0.0)$ & $2(5.7)$ & $5(20.0)$ & $23(85.7)$ & $5(100.0)$ \\
$\quad$ Vancomycin-resistant (67) & $0(0.0)$ & $0(0.0)$ & $2(3.0)$ & $29(46.3)$ & $36(100.0)$ & - \\
Coagulase-negative staphylococi (812) & $65(8.0)$ & $432(61.2)$ & $282(95.9)$ & $31(99.8)$ & $2(100.0)$ & - \\
Viridans group streptococci (24) & $14(58.3)$ & $2(66.7)$ & $7(95.9)$ & $1(100.0)$ & - & - \\
B-haemolytic streptococci (99) & $69(69.7)$ & $27(97.0)$ & $3(100.0)$ & - & - & - \\
\hline
\end{tabular}

infections, especially those in immunosuppressed patients [25]. Although the clinical importance of bactericidal activity in the treatment of most infections remains controversial, antimicrobial treatments that provide bactericidal therapy have been demonstrated to be superior to bacteriostatic regimens in the treatment of $S$. aureus bacteremia/sepsis and also in the treatment of systemic infections in immunosuppressed patients [26,27].

Although vancomycin resistance in enterococci is definitely increasing in the Brazilian hospitals participating in the SENTRY Program, the results of this and other studies indicate that this increase is medical center specific and probably related to clonal dissemination of resistant clones $[6,28,29]$. In Brazil, acquired vancomycin resitance among enterococci was initially reported in E. faecium. However, more recently $E$. faecalis became the predominant vancomycinresistant Enterococcus species in Brazilian hospitals. The clonality of VRE has been evaluated in medical center (048) in a previously published study which found two predominant clones among vancomycin-resistant $E$. faecalis and a greater clonal variability among vancomycin-resistant $E$. faecium isolated in the intensive care units of that hospital [30].

The prevalence of vancomycin-resistant $E$. faecalis has increased continuously, but is essentially restrict to one medical center (048). Regarding E. faecium, the results of this study showed that the prevalence of infections caused by $E$. faecium in general (vancomycin-susceptible or resistant) remained very low until vancomycin-resistant $E$. faecium emerged. E. faecium represented only 1.7 and 5.3\% of enterococcal strains in medical centers where vancomycinresistant E. faecium was not observed, and only $3.6 \%$ in center 101 in the 2005-2007 period (vancomycin-resistant $E$. faecium emerged in 2008 in this medical center). In contrast, E. faecium represented $35.5 \%$ of enterococcal strains collected in medical center 048 in the period of this study and $27.4 \%$ of enterococcal strains isolated in medical center 101 in 2008. In summary, the occurrence of E. faecium infections increased drastically when vancomycin-resistant 
E. faecium emerged and the majority of E. faecium infections are due to vancomycin-resistant strains.

Only two compounds showed acceptable activity against enterococci, those being daptomycin and linezolid. Linezolid had potent in vitro activity against vancomycin-resistant $E$. faecalis and E. faecium, as well as good therapeutic efficacy for VRE bacteremia in mice [25]. However, as previously discussed, linezolid has some limitations due to its predominantly bacteriostatic activity [25].

In general, daptomycin demonstrated excellent in vitro activity against recent clinical isolates of Gram-positive species (3,907 isolates). Daptomycin is a novel lipopeptide with potent in vitro activity against Gram-positive cocci [3135]. Daptomycin has a unique mechanism of action and has demonstrated a rapid bactericidal activity against a wide spectrum of Gram-positive organisms, including multidrugresistant (MDR) strains of staphylococci, enterococci and streptococci [36]. Furthermore, daptomycin monotherapy was shown to be superior to vancomycin monotherapy in the treatment of experimental endocarditis due to methicillin (oxacillin)-resistant S. aureus (MRSA)[26]. This agent was approved by the USA Food and Drug Administration (USAFDA) and by the European Medicine Agency for the treatment of complicated SSSI using a dose of $4 \mathrm{mg} / \mathrm{kg}$ every 24 hours and $S$. aureus bacteremia, including right-sided endocarditis, at an increased dose of $6 \mathrm{mg} / \mathrm{kg}$ every 24 hours [31,37].

Although vancomycin resistance rates in Brazil appears to be relatively low compared to those reported in the USA [7], VRE has emerged and rapidly disseminated in some medical centers. Furthermore, the results of the present study confirmed previous reports by showing that daptomycin is active against many MDR Gram-positive strains and that vancomycin resistance does not significantly affect its in vitro activity.

\section{References}

1. NNIS. National Nosocomial Infections Surveillance (NNIS) Systems Report. 2004. Data summary from January 1992 through June 2004, issued October 2004. Am J Infect Control 2004;32:470-85.

2. Sader H.S., Jones R.N., Gales A.C., et al. SENTRY antimicrobial surveillance program report: Latin American and Brazilian results for 1997 through 2001. Braz J Infect Dis 2004;8:25-79.

3. Deresinski S. Methicillin-resistant Staphylococcus aureus: An evolutionary, epidemiologic, and therapeutic odyssey. Clin Infect Dis 2005; $40: 562-73$.

4. Rice L.B. Antimicrobial resistance in Gram-positive bacteria. Am J Med 2006;119:S11-9; discussion S62-70.

5. Casey A.L., Lambert P.A., Elliott T.S. Staphylococci. Int J Antimicrob Agents 2007;29 Suppl 3:S23-32.

6. Tacconelli E., Cataldo M.A. Vancomycin-resistant enterococci (VRE): transmission and control. Int J Antimicrob Agents 2008;31:99-106.

7. Deshpande L.M., Fritsche T.R., Moet G.J., et al. Antimicrobial resistance and molecular epidemiology of vancomycin-resistant enterococci from North America and Europe: a report from the SENTRY antimicrobial surveillance program. Diagn Microbiol Infect Dis 2007;58:163-70.

8. Biedenbach D.J., Moet G.J., Jones R.N. Occurrence and antimicrobial resistance pattern comparisons among bloodstream infection isolates from the SENTRY Antimicrobial Surveillance Program (1997-2002). Diagn Microbiol Infect Dis 2004;50:59-69.
9. Andrade S., Sader H.S., Barth A., et al. Antimicrobial susceptibility of Gram-negative bacilli isolated in Brazilian hospitals participating in the SENTRY Program (2003-2008). Braz J Infect Dis 2008;12(Suppl 2):3-9).

10. Clinical and Laboratory Standards Institute. M7-A7, Methods for dilution antimicrobial susceptibility tests for bacteria that grow aerobically; approved standard - seventh edition. Wayne, PA: CLSI, 2006.

11. Clinical and Laboratory Standards Institute. M100-S18, Performance standards for antimicrobial susceptibility testing, 18th informational supplement. Wayne, PA: CLSI, 2008.

12. Muto C.A., Jernigan J.A., Ostrowsky B.E., et al. SHEA guideline for preventing nosocomial transmission of multidrug-resistant strains of Staphylococcus aureus and enterococcus. Infect Control Hosp Epidemiol 2003;24:362-86.

13. Hsueh P.R., Chen W.H., Teng L.J., Luh K.T. Nosocomial infections due to methicillin-resistant Staphylococcus aureus and vancomycin-resistant enterococci at a university hospital in Taiwan from 1991 to 2003: resistance trends, antibiotic usage and in vitro activities of newer antimicrobial agents. Int $\mathrm{J}$ Antimicrob Agents 2005;26:43-9.

14. Tenover F.C., Moellering R.C., Jr. The rationale for revising the Clinical and Laboratory Standards Institute vancomycin minimal inhibitory concentration interpretive criteria for Staphylococcus aureus. Clin Infect Dis 2007;44:1208-15.

15. Gales A.C., Sader H.S., Andrade S.S., et al. Emergence of linezolidresistant Staphylococcus aureus during treatment of pulmonary infection in a patient with cystic fibrosis. Int $\mathrm{J}$ Antimicrob Agents 2006;27:300-2.

16. Sader H.S., Jones R.N., Ballow C.H., et al. Antimicrobial susceptibility of quinupristin/dalfopristin tested against Grampositive cocci from Latin America: results from the global SMART (GSMART) surveillance study. Braz J Infect Dis 2001;5:21-31.

17. Haroche J., Morvan A., Davi M., et al. Clonal diversity among streptogramin A-resistant Staphylococcus aureus isolates collected in French hospitals. J Clin Microbiol 2003;41:586-91.

18. Ibrahim E.H., Sherman G., Ward S., et al. The influence of inadequate antimicrobial treatment of bloodstream infections on patient outcomes in the ICU setting. Chest 2000;118:146-55.

19. Hughes W.T., Armstrong D., Bodey G.P., et al. 2002 guidelines for the use of antimicrobial agents in neutropenic patients with cancer. Clin Infect Dis 2002;34:730-51.

20. Kirst H.A., Thompson D.G., Nicas T.I. Historical yearly usage of vancomycin. Antimicrob Agents Chemother 1998;42:1303-4.

21. Sader H.S., Fritsche T.R., Jones R.N. Daptomycin bactericidal activity and correlation between disk and broth microdilution method results in testing of Staphylococcus aureus strains with decreased susceptibility to vancomycin. Antimicrob Agents Chemother 2006;50:2330-6.

22. Sader H., Jones R., Rybak M. Evaluation of the accuracy of the Etest macromethod for detection of heterogeneous vancomycinintermediate $S$. aureus (hVISA) strains. 48th International Conference on Antimicrobial Agents and Chemotherapy (ICAAC); Washington, D.C.: American Society for Microbiology 2008.

23. Sakoulas G., Moise-Broder P.A., Schentag J., et al. Relationship of MIC and bactericidal activity to efficacy of vancomycin for treatment of methicillin-resistant Staphylococcus aureus bacteremia. J Clin Microbiol 2004;42:2398-402.

24. Soriano A., Marco F., Martinez J.A., et al. Influence of vancomycin minimum inhibitory concentration on the treatment of methicillin-resistant Staphylococcus aureus bacteremia. Clin Infect Dis 2008;46:193-200.

25. Moellering R.C. Linezolid: the first oxazolidinone antimicrobial. Ann Intern Med 2003;138:135-42.

26. Pankey G.A., Sabath L.D. Clinical relevance of bacteriostatic versus bactericidal mechanisms of action in the treatment of Grampositive bacterial infections. Clin Infect Dis 2004;38:864-70. 
27. Sakoulas G., Eliopoulos G.M., Alder J., Eliopoulos C.T. Efficacy of daptomycin in experimental endocarditis due to methicillinresistant Staphylococcus aureus. Antimicrob Agents Chemother 2003; 47:1714-8.

28. Cordeiro J.C., Silbert S., Reis A.O., Sader H.S. Inter-hospital dissemination of glycopeptide-resistant Enterococcus faecalis in Brazil. Clin Microbiol Infect 2004;10:260-2.

29. Mascini E.M., Bonten M.J. Vancomycin-resistant enterococci: Consequences for therapy and infection control. Clin Microbiol Infect 2005;11 Suppl 4:43-56.

30. d’Azevedo P.A., Furtado G.H., Medeiros E.A., et al. Molecular characterization of vancomycin-resistant Enterococci strains eight years apart from its first isolation in Sao Paulo, Brazil. Rev Inst Med Trop Sao Paulo 2008;50:195-8.

31. Fowler V.G., Jr., Boucher H.W., Corey G.R., et al. Daptomycin versus standard therapy for bacteremia and endocarditis caused by Staphylococcus aureus. N Engl J Med 2006;355:653-65.
32. Hair P.I., Keam S.J. Daptomycin: A review of its use in the management of complicated skin and soft-tissue infections and Staphylococcus aureus bacteraemia. Drugs. 2007;67:1483-512.

33. Levine D.P., Lamp K.C. Daptomycin in the treatment of patients with infective endocarditis: Experience from a registry. Am J Med 2007;120:S28-33.

34. Sader H.S., Jones R.N. The activity of daptomycin against wild-type Staphylococcus aureus and strains with reduced susceptibility to vancomycin. Clin Infect Dis 2006;43:798-9; author reply 99-800.

35. Sauermann R., Rothenburger M., Graninger W., Joukhadar C. Daptomycin: a review 4 years after first approval. Pharmacology 2008;81:79-91.

36. Sader H.S., Streit J.M., Fritsche T.R., Jones R.N. Antimicrobial activity of daptomycin against multidrug-resistant Gram-positive strains collected worldwide. Diagn Microbiol Infect Dis 2004; 50:201-4.

37. Package Insert. Cubicin (daptomycin for injection). Lexington MA. (Cubist Pharmaceuticals, Inc). Available at http://www.cubicin.com/ pdf/PrescribingInformation.pdf. Accessed on January 13, 2008. 